\title{
The Latest Strategy for Keloid and Hypertrophic Scar Prevention and Treatment: The Nippon Medical School (NMS) Protocol
}

\author{
Rei Ogawa, Teruyuki Dohi, Mamiko Tosa, \\ Masayo Aoki and Satoshi Akaishi
}

\begin{abstract}
Department of Plastic, Reconstructive and Aesthetic Surgery, Nippon Medical School Hospital, Tokyo, Japan In 2006, we established a scar/keloid-specialized unit in the Department of Plastic, Reconstructive, and Aesthetic Surgery at Nippon Medical School (NMS) in Tokyo, Japan. In the ensuing 15 years, we treated approximately 2,000 new scar/keloid patients annually. This extensive experience has greatly improved the efficacy of the treatments we offer. Therefore, we discuss here the latest NMS protocol for preventing and treating keloids and hypertrophic scars. While this protocol was optimized for Japanese patients, our experience with a growing body of non-Japanese patients suggests that it is also effective in other ethnicities. The extensive evidence-based experience underlying the NMS protocol suggests that it may be suitable as the foundation of a standard international prevention/treatment algorithm for pathological scars. (J Nippon Med Sch 2021; 88: 2-9)
\end{abstract}

Key words: keloid, hypertrophic scar, wound

\section{Introduction}

In 2006, we established a scar/keloid-specialized unit in the Department of Plastic, Reconstructive, and Aesthetic Surgery at Nippon Medical School (NMS) in Tokyo, Japan. In the following 15 years, we treated approximately 2,000 new scar/keloid patients annually. This vast experience has greatly improved the efficacy of the treatments we offer. Therefore, we discuss here the latest NMS algorithm for preventing and treating keloids and hypertrophic scars (HSs). While this algorithm has been optimized for Japanese patients, our experiences with a growing body of international patients suggest that it is also effective in other ethnicities and could therefore serve as the starting point in the development of an international prevention/treatment algorithm.

\section{Pathomechanisms of Keloids/HSs}

To effectively prevent and treat keloids/HSs, it is necessary to understand the pathomechanisms that drive their formation and progression. The abnormal growth of these scars is powered by chronic inflammation in the reticular dermis ${ }^{1}$ that is triggered by dermal injury or irri- tation, including trauma, burn, surgery, vaccination, skin piercing, acne, and herpes zoster. In general, the scars that spontaneously lose this dermal inflammation over time are defined as HSs, whereas the scars with enduring inflammation are defined as keloids. This difference in inflammation durability explains the disparate growth habits of HSs and keloids. Thus, the temporary, waning inflammation in HSs means that they do not grow over the borders of the original wound. By contrast, the continuous, escalating inflammation of keloids causes them to grow into the adjacent normal skin.

In terms of histopathology, the inflammation and the resulting accumulation of extracellular matrix causes dermal nodules and thick eosinophilic (hyalinizing) collagen bundles called "keloidal collagen" to form over time ${ }^{2}$. If the dermal nodule is the main histological finding, it is diagnosed as an HS. This suggests that keloids/HSs are manifestations of the same inflammatory fibroproliferative condition and simply differ in the intensity and duration of inflammation.

Multiple lines of evidence suggest that the intensity/ duration of dermal inflammation in keloids/HSs is dic-

Correspondence to Rei Ogawa, MD, PhD, FACS, Department of Plastic, Reconstructive and Aesthetic Surgery, Nippon Medical School, 1-1-5 Sendagi, Bunkyo-ku, Tokyo 113-8603, Japan

E-mail: r.ogawa@nms.ac.jp

https://doi.org/10.1272/jnms.JNMS.2021_88-106

Journal Website (https://www.nms.ac.jp/sh/jnms/) 
tated by genetic, systemic, local, and lifestyle risk factors ${ }^{1}$. Genetic causes include single nucleotide polymorphisms $^{3,4}$ and genetic diseases such as Rubinstein-Taybi syndrome $e^{5,6}$. There are probably many other, as yet unidentified, genetic factors. Systemic factors include pregnancy $^{7}$, hypertension ${ }^{8}$, high circulating concentrations of inflammatory cytokines ${ }^{9}$, and possibly female sex ${ }^{10}$.

The key local risk factor is mechanical force on the wound/scar: considerable evidence shows that this strongly influences inflammation-driven pathological scarring $^{11-13}$. Indeed, our finite-element analysis ${ }^{13}$ suggests that keloid shape is largely determined by the direction of the tension on the skin around the wound/scar site. We also showed that keloids are particularly prevalent on body regions with strong skin-stretching force due to body movements, such as the joints, anterior neck, chest wall, scapula/upper-arm, and suprapubic region. Repeated dermal injury and infection is also a local risk factor.

Lifestyle risk factors include strong/continuous body movements (e.g. those of athletes/manual workers) that stretch the wounds/scars. Other factors are excessive consumption of hot/spicy foods and alcohol and overlong bathing in hot water, which may augment wound/ scar inflammation. Obesity may also be a risk factor.

\section{Prevention of Keloids/HSs}

Because traumatic, surgical, and burn wounds and wounds caused by infectious agents, can turn into pathological scars, it is important to assess the pathological scarring risk of patients with such wounds. If the patient or his/her family has a history of keloid and/or the wound is on a high-risk zone, the patient should be considered to be high risk and treated appropriately, as described below.

\section{Trauma and Burn Wounds with a Raw Surface}

In general, the longer wounds take to epithelialize, the greater the risk that keloids/HSs develop. Therefore, trauma/burn wounds should undergo primary suturing or skin grafting/flap transfer as soon as possible. However, if surgery cannot be planned, the raw surface must be encouraged to epithelialize rapidly by applying conservative therapies that limit infection and inflammation and accelerate granulation-tissue formation. These therapies, which should be applied on a case-by-case basis, include negative-pressure wound therapy, appropriate wound dressing materials, topical drugs (e.g. basic fibroblast growth factor spray), antibiotics, and antiinflammatory agents. The wound should also be stabi- lized by placing bandages or garments over the dressing.

In normal wound healing, once epithelialization is completed, the inflammation in the dermis/dermal-like granulation tissues under the epidermis starts to subside spontaneously and the dermal tissues begin maturing. However, if strong/repetitive mechanical forces are exerted on the immature scar, they can prolong the inflammation, thereby fomenting pathological scarring. One of the keys to preventing such scarring is to understand that the healing rates of the epidermis and dermis differ completely. In the case of sutured wounds, the epidermis can regenerate within 7-10 days, leading both the patient and the physician to believe that the wound has healed completely. In fact, it can take 3 months before the dermis regains $90 \%$ of its normal strength ${ }^{14}$. This lengthy vulnerability to inflammation-triggering mechanical forces means that it is essential to provide the immature scar with protracted external mechanical support (i.e. scar stabilization) until maturity has been attained (i.e. the scar becomes soft, pliable, and flat).

Therefore, all immature scars should be fixed continuously with tape. Silicone tape is the best choice. The tape is retained until it detaches naturally. Alternatively, silicone-gel sheets or cheaper paper tapes can be used on a case-by-case basis.

When an individual is at high risk of keloid formation and/or the scars are located on keloid-prone regions, scar stabilization should be prolonged for at least 6-12 months after maturation. It should also be combined with first steroid ointment and then tape/plaster administration. In Japan, two types of steroid tapes are avail$a^{15} e^{15}$ : the strong deprodone-propionate plaster (Eclar ${ }^{\circledR}$ plaster; Hisamitsu Pharmaceutical Co., Inc., Tokyo, Japan) should be used for adults while the weak fludroxycortide tape (Drenison ${ }^{\circledR}$ tape; Teikoku Seiyaku Co., Inc., Kagawa, Japan) is suitable for children. The steroid ointment should be applied for the first 2-3 weeks after epithelialization because the epidermis is still immature (full epithelialization takes 4 weeks) and could be injured by daily steroid tape/plaster changes. Once steroid tape/ plaster replaces the ointment, it is continued until the scar is soft and flat. Scar redness should be ignored: continuing steroid tape/plaster just because of redness elevates the risk of telangiectasia because the steroid thins the structures supporting the blood vessels. Once steroid tape/plaster is discontinued, a non-steroidal ointment/ cream such as heparinoid ointment/lotion/spray (Hirudoid $^{\circledR}$; Maruho Co., Inc., Osaka, Japan) or an NSAID ointment/cream (Ibuprofen piconol: Staderm ${ }^{\circledR}$; Torii 
Pharmaceutical Co., Ltd., Tokyo, Japan) should be applied to keep scar surface moist and inhibit inflammation.

In the case of low-risk individuals and/or wounded regions (e.g. the face, lower leg), wound stabilization is sufficient to prevent pathological scarring.

\section{Primarily Closed Wounds}

Sutured wounds should be cleaned daily with tap water/saline or disinfectant and covered with nonadhesive materials until the sutures can be removed. This prevents infections and wound stretching, thereby blocking excessive inflammation. After suture removal, wound stabilization with silicone tape/gel-sheets should be started. For high-risk individuals and/or regions, silicone-tape fixation should be replaced 2-3 weeks later with steroid tape/plaster that continues until scar maturation. For low-risk individuals and/or regions, siliconetape fixation is sufficient.

\section{Inflammatory Skin Diseases}

Inflammation associated with skin diseases such as acne, folliculitis, and herpes zoster can strongly provoke pathological scarring if it spreads into the dermis. To prevent this, the lesions caused by these diseases should be treated topically with ointments/creams that contain anti-infection agents until the exudation stops. The antiinflammatory preventive measures described above should then be started. Thus, high-risk individuals and/ or regions should be treated with silicone tape-mediated wound stabilization plus 2-3-week steroid ointment treatment that is then replaced by steroid tape/plaster. Wound stabilization alone is sufficient for low-risk individuals and/or regions.

\section{Early Detection of Keloids/HSs}

The earlier pathological scarring is detected, the easier it is to treat it. Therefore, at follow-up after epithelialization, scars should be palpated. Any stiffness/hardness, even in a small part of the scar, indicates continued inflammation that has led to collagen accumulation. In this case, silicone-tape fixation should be changed immediately to steroid tape/plaster. If it is ineffective in the next 1-3 months, steroid injections should be started/added. If the scar remains refractory, surgery and adjuvant therapy is indicated.

In general, keloids/HSs form 1-3 months after injury/ surgery. Therefore, patients should be instructed to touch their scar daily for at least 3 months to check for stiffness.

\section{Treatment of Hypertrophic Scars and Keloids Diagnosis of Keloid/HS Risk}

The Japan Scar Workshop (JSW) scar scale (JSS) ${ }^{16}$ helps to differentially diagnose HS/keloid-like lesions. It also has indicators that suggest the scar may be or become intractable:

1. The patient has multiple keloid/HS-like lesions on the body

2. The keloid/HS-like lesion is large, even if it is solitary

3. Age at pathological-scar onset is $<20$ years

4. The patient is a female around 10-30 years of age ( $i$. e. of reproductive age)

5. The patient is pregnant

6. The patient has hypertension

7. The patient is a physical laborer/professional athlete

8. The lesion is on a mobile body area (e.g. major joints, anterior chest/neck)

If the patient is judged to have low risk, his/her lesion resembles an HS and conservative therapy is the primary choice. However, if contracture is observed, especially on a joint, contracture-releasing surgery may be indicated. After surgery, recurrence can be prevented by steroid tape/plaster.

If the patient is judged to have high risk, his/her lesion resembles a keloid and multimodal therapy should be planned. If surgery is selected, postoperative radiotherapy must be performed to prevent recurrence.

\section{Steroid Tape/plaster}

Steroid tape/plaster ${ }^{15}$ is the first-line therapy for keloids/HSs in the NMS protocol (Fig. 1). Most pediatric and older patients can be treated by steroid tape/plaster alone because they have much thinner skin, which easily absorbs the steroid. However, thick keloids are difficult to treat by steroid tape/plaster alone and require the addition of corticosteroid injections (Kenacort ${ }^{\circledR}$; BristolMyers Squibb K.K., Tokyo, Japan). These injections cause the keloids/HSs to soften, thereby enhancing the absorption of steroid from the tape/plaster.

Steroid tape/plaster also serves to prevent keloid/HS recurrence after excision. The same protocol used to prevent keloids/HSs after skin injury/infection is used: thus, steroid tape/plaster is started with daily changes 23 weeks after suture removal; scar stiffness is checked during follow-up; steroid injection is added if the scar is refractory to the tape/plaster; and tape/plaster therapy is continued until the scar softens and flattens. Treatment concludes with non-steroidal ointment/cream application. 


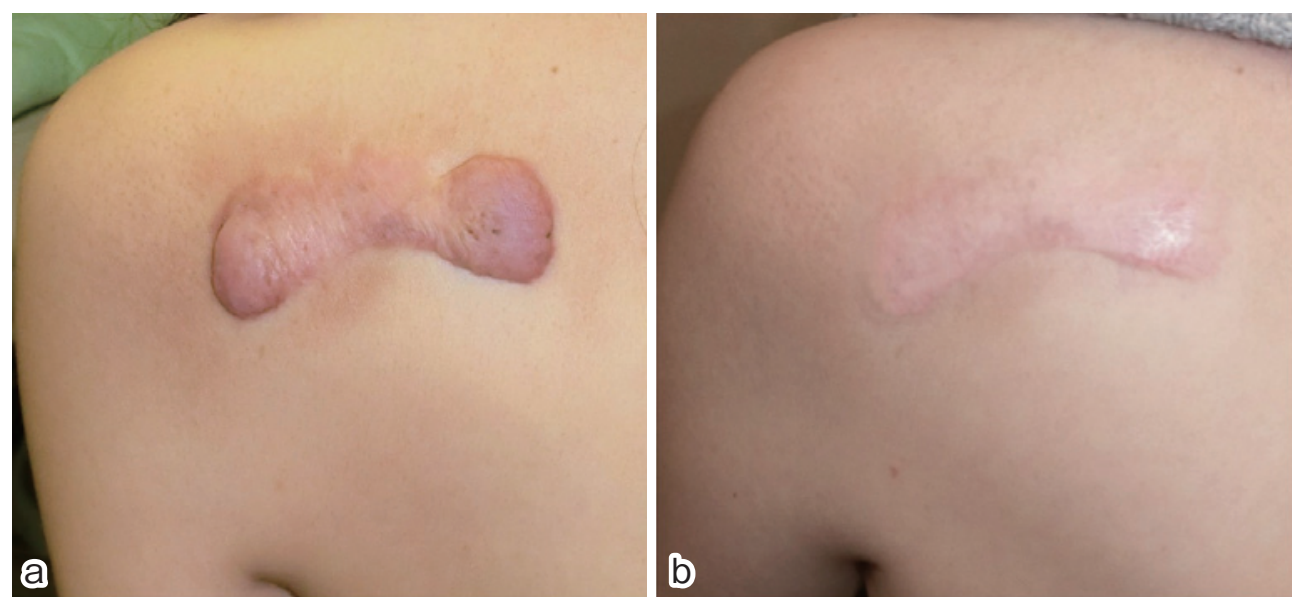

Fig. 1 The left scapular keloid of a woman in her 30s was treated by deprodone-propionate plaster.

(a) Pretreatment.

(b) Twenty-four months after starting treatment.

The tape was placed on the keloid 24 hours a day and changed daily. After 24 months, the inflammation had completely resolved and the subjective and objective symptoms improved.
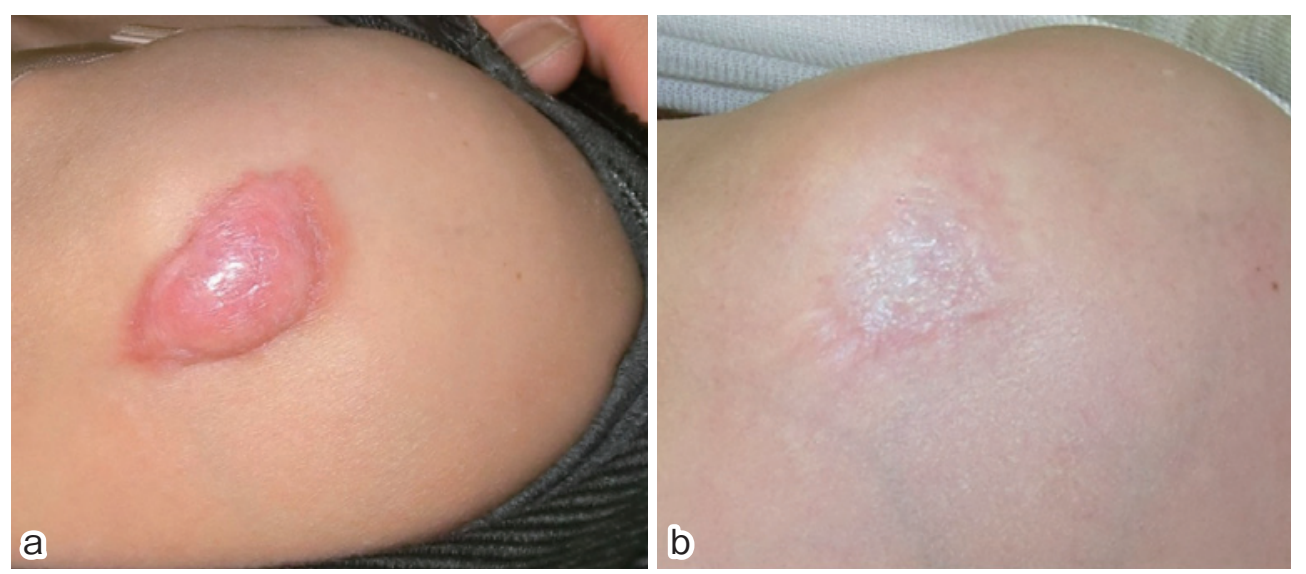

Fig. 2 The right shoulder keloid of a woman in her 50s was treated by triamcinolone acetonide steroid injections.

(a) Pretreatment.

(b) Twenty-four months after starting treatment.

The keloid underwent steroid injection (5 mg triamcinolone diluted with $1.5 \mathrm{~mL}$ xylocaine $1 \%$ with epinephrine) every 2-3 months. After 24 months, the subjective and objective symptoms improved.

\section{Steroid Injection}

Triamcinolone acetonide is a powerful tool because it rapidly reduces inflammation (Fig. 2). Each injection should comprise 5-10 mg mixed with a local anesthetic such as xylocaine $1 \%$ with epinephrine ${ }^{16}$. Women may experience fewer menstrual irregularities if the dose does not exceed $5 \mathrm{mg}$. We use thin needles such as $30 \mathrm{G}$ and $27 \mathrm{G}$ along with syringes with locks. The initial target of the injection should not be the center of the mass: its hardness means the injection fluid will not disperse suffi- ciently throughout the tissue. The consequent local pressure may also cause pain. Rather, the target should be the scar border where it meets the normal skin: the needle should penetrate either shallowly to target the heavily inflamed leading edge of the scar or deeply towards the center (the scar tissue along this deep plane is softer than the upper central core). Once one or more injections have softened the scar, the needle can be injected straight into the core. Smaller keloids/HSs can improve markedly after just 1-2 injections. If these improvements can be 

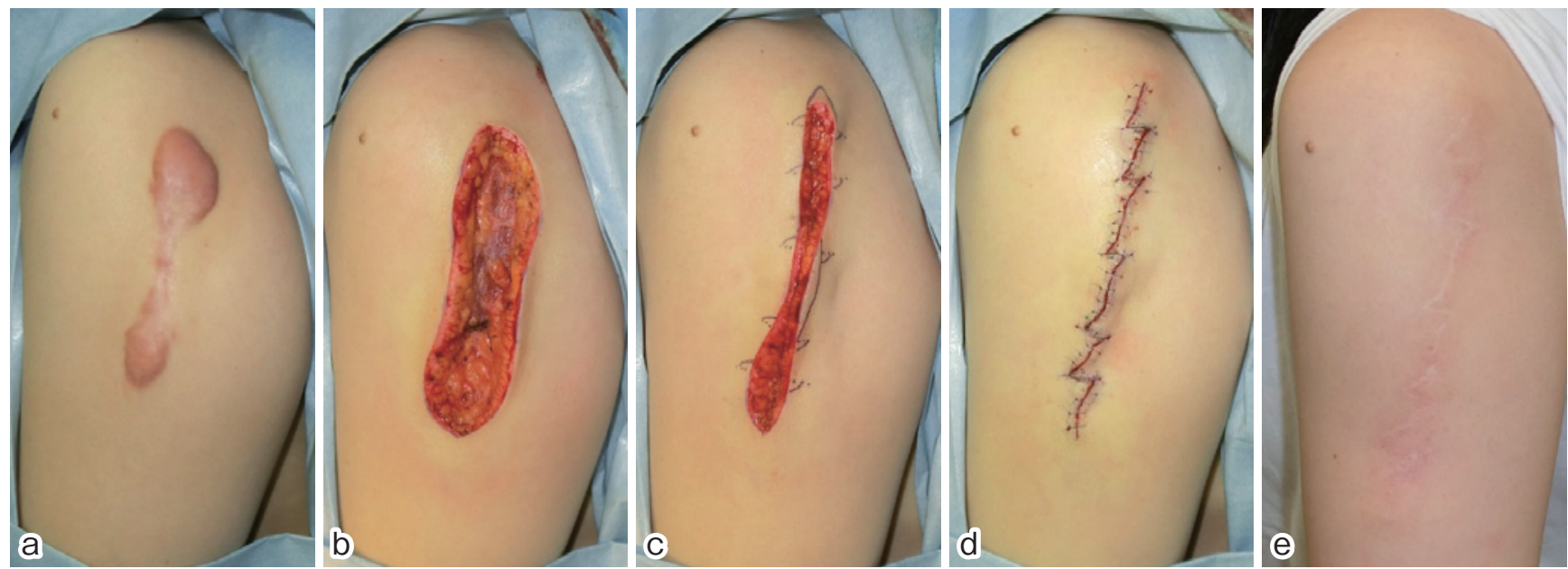

Fig. 3 The left upper-arm keloid of a woman in her 30s was treated by z-plasty and postoperative radiotherapy.

(a) Before surgery.

(b) After keloid excision.

(c) Z-plasty design.

(d) Immediately after surgery.

(e) Two years after surgery.

The keloid developed from BCG vaccination. It was excised, tension was released by fascial sutures, and z-plasties were performed. Postoperative radiotherapy (18 Gy/3 fr/3days) was then conducted. No recurrence was observed 2 years after surgery.

continued/maintained with steroid tape/plaster, further injections are unnecessary. If patients are afraid of injection-induced pain, we recommend steroid tape/plaster until the scar has softened. Injections can then be added because the risk of pain is much reduced.

\section{Surgery}

Surgical monotherapy associates with an atrocious rate of keloid/HS recurrence. Therefore, surgical techniques that abrogate dermal tension, namely, subcutaneous/fascial tensile-reduction sutures, z-plasties, and local flap transfer ${ }^{17,18}$, should be used in combination with postoperative radiation (described below) and steroid tape/ plaster.

Placing sutures on the superficial/deep fascia below the dermis causes the wound edges to juxtapose naturally. This allows dermal sutures to be placed with very little tension, after which superficial sutures can be added. It is essential to realize that without the deep sutures, dermal sutures not only fail to reduce the dermal tension, they may in fact exacerbate it.

Zig-zag sutures, including z-plasties, are good for releasing linear scar contractures and tension (Fig. 3). Another advantage is that segmented scars mature faster than long linear scars. Zig-zag incision/suturing is particularly indicated if scars cross a joint.

Local flaps are also useful for releasing scar contractures (Fig. 4): they expand naturally after surgery and are therefore not prone to postsurgical contractures, unlike skin grafts, which often form pathological scars around the graft. Compared to island flaps, skin-pedicled flaps are superior because they extend more postsurgically and therefore release contractures more effectively ${ }^{19}$. Flap surgery is especially suitable for severe keloids; however, multimodal therapy should be applied to both the excision and donor sites to prevent recurrent and new lesions from forming.

\section{Radiation}

Post-excision keloid/HS recurrence can be effectively controlled by adjuvant radiation. Radiation protocols are constantly improving. In the past, orthovoltage or superficial or soft X-rays were used. However, the safety and efficacy of radiotherapy protocols have improved markedly recently; therefore, we now routinely use postoperative electron-beam irradiation after keloid/HS resection. This, when combined with the surgical and steroid tape/ plaster modalities described above, markedly reduces the recurrence rate: the keloid-recurrence rate after surgery is now less than $10 \%$ in our hospital ${ }^{18}$. Moreover, 15 years of close monitoring and long-term follow-up has shown that this protocol has no major side effects (Fig. 3, 4) ${ }^{18}$.

Another, increasingly popular, radiation modality for keloids is high-dose rate-superficial brachytherapy. Depending on the shape of the surgical scar, an applicator can be used to ensure the evenness and localization of the radiation to the wound surface. However, we prefer electron beams because they reach the reticular dermis 

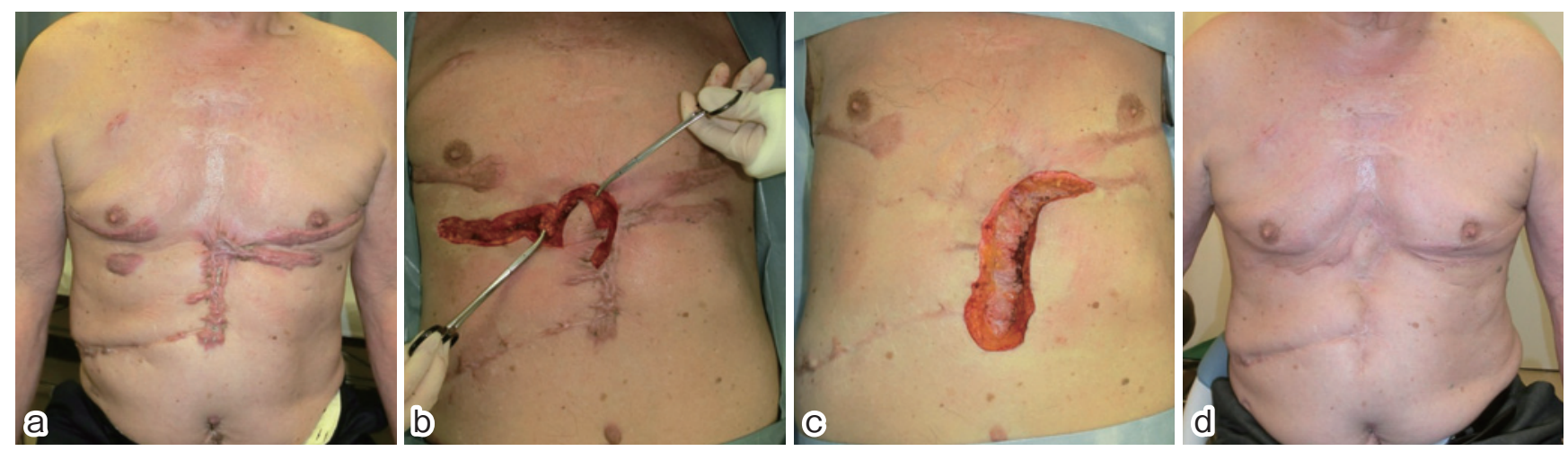

Fig. 4 The chest and abdominal keloids of a man in his 70s were treated by flap surgery, z-plasties, and postoperative radiotherapy.

(a) Before surgery.

(b) Intraoperative view of the first operation, during which the chest keloid was excised and reconstructed with a flap.

(c) Intraoperative view of the second operation, during which the abdominal keloid was removed and closed with z-plasties.

(d) Two years after the second operation.

The keloids were removed in two operations. The donor and recipient sites were irradiated. The post-treatment course was uneventful. After 2 years, recurrence was not observed and the remaining pathological scars had matured.
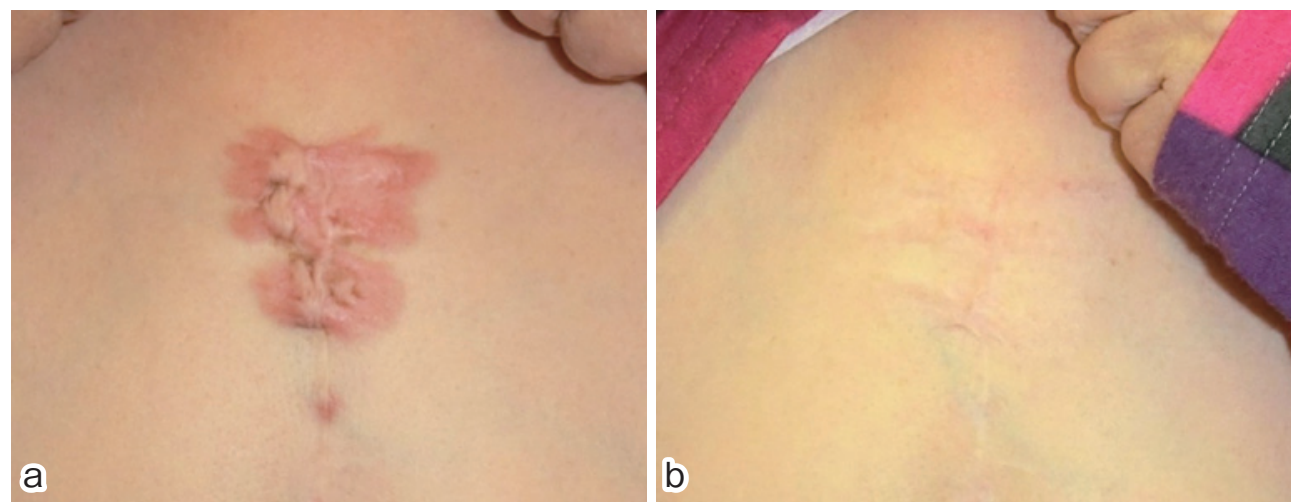

Fig. 5 The chest-wall keloid of a woman in her 70s was treated by radiation monotherapy.

(a) Before treatment.

(b) Eighteen months after treatment.

This mild chest-wall keloid was treated by high-dose rate-superficial brachytherapy (25 Gy/5 fr/5days). The inflammation resolved completely. Six months later, the subjective and objective symptoms improved dramatically. Eighteen months later, the pathological scars matured.

more selectively; moreover, the beam attenuates after passing through the skin and thus has little effect on the internal organs.

To further improve the safety of our adjuvant radiotherapy protocol, we optimized them in 2013 such that fewer fractions are now used ${ }^{18}$. As a result, highrecurrence keloid-excision sites are now treated with 18 Gy/3 fr/3days while low-recurrence sites (e.g. earlobe) and other body sites are now treated with $8 \mathrm{~Gy} / 1 \mathrm{fr} / 1$ day and $15 \mathrm{~Gy} / 2 \mathrm{fr} / 2$ days, respectively.

We also occasionally employ radiation monotherapy to treat older patients or patients with huge keloids (Fig. $5)^{1}$. However, since the total radiation dose needed is higher than the postoperative radiation doses, the radiation should be delivered carefully to avoid secondary radiation carcinogenesis. It is also essential to secure informed consent. However, the risks of primary radiotherapy should be measured against its immense benefits: it rapidly decreases subjective symptoms such as pain and itch and, over the following year, progressively normalizes scar color and thickness.

\section{Laser Therapy}

In our institution, a 1,064-nm long-pulsed Nd:YAG laser is used for flattish keloids/HSs that have low levels of inflammation ${ }^{20,21}$. It is mainly used to decrease the redness/telangiectacia caused by steroid therapy. Flat 
keloids/HSs are particularly suitable for laser therapy because the laser beam can reach the blood vessels in the dermis.

Once scar inflammation has been completely quelled by other methods, fractional abrative lasers can be used for low-risk individuals. This induces a wound healing response that increases collagen III production, thereby encouraging scar remodeling. Note: it is important to cool the skin when conducting laser therapy to prevent superficial injury.

However, these lasers have only limited efficacy with thick, highly inflamed keloids/HSs.

\section{Make-up Therapy}

Medical make-up techniques such as "Rehabilitation Make-up ${ }^{\circledR \prime}$ are recommended if the scars are on exposed areas (e.g. the face, neck, and upper limbs). These techniques can be learned and self-applied by the patients. Since our cosmetics are water-proof, they are not affected by humid/wet conditions. These techniques associate with improved mental health, acceptance of the scar appearance, and more positive attitudes to scar treatment.

Since it is difficult to improve the aesthetics of highly elevated scars with make-up therapy, scar thickness should be reduced with steroid plaster/injection before commencing make-up therapy.

Slightly rough scars can be covered by thin tapes prior to applying the foundation.

\section{Follow-up of Keloids/HSs}

Keloid/HS patients who receive multimodal therapy should undergo prolonged follow-up and be taught how to manage their scar and any new wounds. Patient education is a hugely important factor that dictates the success of our protocol.

\section{Conclusions}

Multimodal therapy, particularly surgery, radiation, and steroid tape/plaster, successfully manages keloids/HSs. Our extensive experience, including with international patients, suggests that the NMS protocol may be suitable as a foundation for a standard international prevention/ treatment algorithm for pathological scars. It should be noted that the NMS protocol relies on close, prolonged follow-up, which is only feasible if keloid/HS treatments are covered by a national universal insurance system (as it is in Japan $)^{22}$. We expect that treatments will improve significantly as our understanding of scar biology grows, more good-quality institutional trials are conducted, and new agents are found.
Conflict of Interest: The authors declare no conflicts of interest.

\section{References}

1. Ogawa R. Keloid and hypertrophic scars are the result of chronic inflammation in the reticular dermis. Int J Mol Sci. 2017;18(3):606.

2. Matsumoto NM, Peng WX, Aoki M, et al. Histological analysis of hyalinised keloidal collagen formation in earlobe keloids over time: collagen hyalinisation starts in the perivascular area. Int Wound J. 2017;14(6):1088-93.

3. Nakashima M, Chung S, Takahashi A, et al. A genomewide association study identifies four susceptibility loci for keloid in the Japanese population. Nat Genet. 2010 Sep;42(9):768-71.

4. Ogawa R, Watanabe A, Than Naing B, et al. Associations between keloid severity and single-nucleotide polymorphisms: importance of rs8032158 as a biomarker of keloid severity. J Invest Dermatol. 2014 Jul;134(7):2041-3.

5. van de Kar AL, Houge G, Shaw AC, et al. Keloids in Rubinstein-Taybi syndrome: a clinical study. Br J Dermatol. 2014;171(3):615-21.

6. Yagi Y, Kuwatsuka Y, Asai M, Honda M, Utani A. Coexistence of keloids and pilomatricoma in a patient with Rubinstein-Taybi syndrome. Dermatol Online J. 2018;24 (1):13030/qt4rq2k5fr.

7. Moustafa MF, Abdel-Fattah MA, Abdel-Fattah DC. Presumptive evidence of the effect of pregnancy estrogens on keloid growth. Case report. Plast Reconstr Surg. 1975 Oct; 56(4):450-3.

8. Arima J, Huang C, Rosner B, Akaishi S, Ogawa R. Hypertension: a systemic key to understanding local keloid severity. Wound Repair Regen. 2015;23(2):213-21.

9. Quong WL, Kozai Y, Ogawa R. A case of keloids complicated by Castleman's disease: Interleukin- 6 as a keloid risk factor. Plast Reconstr Surg Glob Open. 2017;5(5):e 1336.

10. Noishiki C, Hayasaka Y, Ogawa R. Sex differences in keloidogenesis: An analysis of 1659 keloid patients in Japan. Dermatol Ther (Heidelb). 2019;9(4):747-54.

11. Ogawa R, Akaishi S, Huang C, et al. Clinical applications of basic research that shows reducing skin tension could prevent and treat abnormal scarring: the importance of fascial/subcutaneous tensile reduction sutures and flap surgery for keloid and hypertrophic scar reconstruction. J Nippon Med Sch. 2011;78(2):68-76.

12. Ogawa R, Akaishi S, Kuribayashi S, Miyashita T. Keloids and hypertrophic scars can now be cured completely: Recent progress in our understanding of the pathogenesis of keloids and hypertrophic scars and the most promising current therapeutic strategy. J Nippon Med Sch. 2016;83 (2):46-53.

13. Akaishi $\mathrm{S}$, Akimoto M, Ogawa R, Hyakusoku H. The relationship between keloid growth pattern and stretching tension: visual analysis using the finite element method. Ann Plast Surg. 2008;60(4):445-51.

14. Levenson SM, Geever EF, Crowley LV, Oates JF 3rd, Berard CW, Rosen $\mathrm{H}$. The healing of rat skin wounds. Ann Surg. 1965;161(2):293-308.

15. Goutos I, Ogawa R. Steroid tape: A promising adjunct to scar management. Scars Burn Heal. 2017;3 : 2059513117690937.

16. Ogawa R, Akita S, Akaishi S, et al. Diagnosis and treatment of keloids and hypertrophic scars-Japan scar work- 
shop consensus document 2018. Burns Trauma. 2019;7:39.

17. Ogawa R. Surgery for scar revision and reduction: from primary closure to flap surgery. Burns Trauma. 2019;7:7.

18. Ogawa R, Tosa M, Dohi T, Akaishi S, Kuribayashi S. Surgical excision and postoperative radiotherapy for keloids. Scars Burn Heal. 2019;5:2059513119891113.

19. Yoshino Y, Kubomura K, Ueda H, Tsuge T, Ogawa R. Extension of flaps associated with burn scar reconstruction: A key difference between island and skin-pedicled flaps. Burns. 2018;44(3):683-91.

20. Koike S, Akaishi S, Nagashima Y, Dohi T, Hyakusoku H, Ogawa R. Nd:YAG laser treatment for keloids and hypertrophic scars: An analysis of 102 cases. Plast Reconstr Surg Glob Open. 2015;2(12):e272.

21. Tsai CH, Kao HK, Akaishi S, An-Jou Lin J, Ogawa R. Combination of 1,064-nm neodymium-doped yttrium aluminum garnet laser and steroid tape decreases the total treatment time of hypertrophic scars: An analysis of 40 cases of cesarean-section scars. Dermatol Surg. 2019 Nov
5;Forthcoming 2020. doi: 10.1097/DSS.0000000000002235

22. Takura T. An evaluation of clinical economics and cases of cost-effectiveness. Intern Med. 2018;57(9):1191-200.

(Received, May 30, 2020)

(Accepted, July 2, 2020)

(J-STAGE Advance Publication, August 1, 2020)

Journal of Nippon Medical School has adopted the Creative Commons Attribution-NonCommercial-NoDerivatives 4.0 International License (https://creativecommons.org/licenses/by-nc-nd/4.0/) for this article. The Medical Association of Nippon Medical School remains the copyright holder of all articles. Anyone may download, reuse, copy, reprint, or distribute articles for non-profit purposes under this license, on condition that the authors of the articles are properly credited. 